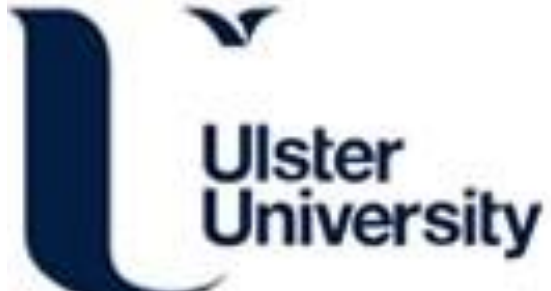

\section{Design and evaluation of a smartphone based wearable life-logging and social interaction system}

Burns, W., Nugent, C., McCullagh, P., \& Zheng, H. (2014). Design and evaluation of a smartphone based wearable life-logging and social interaction system. In Unknown Host Publication (pp. 435 -440). IEEE. https://doi.org/10.1109/CBMS.2014.42

Link to publication record in Ulster University Research Portal

\section{Published in:}

Unknown Host Publication

Publication Status:

Published (in print/issue): 29/05/2014

DOI:

10.1109/CBMS.2014.42

\section{Document Version}

Author Accepted version

\section{General rights}

Copyright for the publications made accessible via Ulster University's Research Portal is retained by the author(s) and / or other copyright owners and it is a condition of accessing these publications that users recognise and abide by the legal requirements associated with these rights.

\section{Take down policy}

The Research Portal is Ulster University's institutional repository that provides access to Ulster's research outputs. Every effort has been made to ensure that content in the Research Portal does not infringe any person's rights, or applicable UK laws. If you discover content in the Research Portal that you believe breaches copyright or violates any law, please contact pure-support@ulster.ac.uk. 


\section{Design and evaluation of a smartphone based wearable life-logging and social interaction system}

\author{
William Burns \\ Computer Science Research Institute \\ University of Ulster \\ Jordanstown, United Kingdom \\ wp.burns@ulster.ac.uk \\ Paul McCullagh \\ Computer Science Research Institute \\ University of Ulster \\ Jordanstown, United Kingdom \\ pj.mccullagh@ulster.ac.uk
}

\author{
Chris Nugent \\ Computer Science Research Institute \\ University of Ulster \\ Jordanstown, United Kingdom \\ cd.nugent@ulster.ac.uk \\ Huiru Zheng \\ Computer Science Research Institute \\ University of Ulster \\ Jordanstown, United Kingdom \\ h.zheng@ulster.ac.uk
}

\begin{abstract}
In this paper we outline the design, development and evaluation of a smartphone based life-logging and social interaction reminder system intended for use by persons with dementia. By using a smartphone, the wearer's daily activities can be recorded in picture format, along with meta data providing activity levels and location data. In addition to this data, social interactions can also be logged and subsequently identified, using Quick Response (QR) codes. The intervention was evaluated on six healthy participants aged between $24-46$ years of age who wore the system for 2.5 hours. The qualitative feedback received was that the technology was easy to use and was responsive and accurate at identifying, recording and displaying social interaction data.
\end{abstract}

Keywords-component; Smartphone, Life-logging, Dementia, Social Interactions, User Interaction.

\section{INTRODUCTION}

The world's population currently exceeds seven billion people and life expectancy is expected to increase sharply in the next 30 years [1]. With this increased life expectancy, age related impairments, both physical and cognitive are also on the increase [2]. These can range from reduced dexterity, diminished hearing and vision and increased prevalence of chronic diseases. Within the next decade, it is predicted that the number of older persons will outnumber the number of persons aged less than five-years old [3]. As a result, an increasing social and economical burden will be placed on healthcare systems [4].

There are many complications associated with increased prevalence of chronic disease; $75 \%$ of older persons have at least one chronic disease with $50 \%$ having at least two long term conditions [5]. Of the chronic diseases, dementia, cancer, diabetes, chronic heart disease and stroke are the most common. On a global scale, dementia has an estimated 35 million sufferers [3]. This number is expected to increase within the next 20 years to approximately 65 million people [6].
In this paper we present an assistive technology, the $\mathrm{R}^{3} \mathrm{~S}$ (Real-time social Reminder and Reminiscence System), that harnesses mobile and wearable technologies to address the unmet needs specifically relating to personalized information, social contact and perceived safety. This work aims to answer the following research question' 'by using technology, is it possible to improve the independence of a person with dementia and enable them to live longer within their own home?".

\section{BACKGROUND}

Dementia is an 'umbrella' term that describes a number of neurological diseases and conditions that results in reduced cognitive function [7].

There are a number of unmet needs associated with persons with dementia (PwD). These include [8]:

- $\quad$ the need for providing personalized information (e.g. appointments, medication reminders etc.).

- the need for support with regards to the symptoms of dementia. Support to enhance participation and supervision/guidance.

- the need for maintaining social contact and company.

- $\quad$ the need for health monitoring safety.

In the following Sections related research associated with life-logging and social interaction prompting is evaluated; the proposed system's technological components are described; and the results of a user evaluation with six participants are presented.

\section{RELATED WORK}

One of the major components of the proposed system is the use of a life-logging system. Life-logging [9] is the process of recording aspects of one's life using technology. The data collected can comprise pictures, audio, movement and physiological data. 
There are a number of commercially available image capturing life-logging devices available. Microsoft have developed the SenseCam [10] which captures an image with a small wearable camera with a fish eye lens. Additional data may be captured using light sensors and accelerometers. All of the images are stored locally on the device's memory card. Doherty et al. used the data recorded from SenseCam to populate a 'SenseCam Browser' that segmented the large dataset recorded into several events within the data [11]. Their software also allowed users to annotate the recorded data.

Memoto is another similar commercially available wearable camera that enables the recording of life-logging data in picture format [12]. It records images every 30 seconds and supplements the data with Global Positioning System (GPS) location information. The recorded data may be analyzed using a smartphone app, which organizes the data into a searchable timeline.

A similar commercial product to the Memoto device is the Autographer [13]. This wearable camera records images and supplementary data for review later on an iOS device. This supplementary data is harnessed from five in-built sensors; accelerometer, magnetometer, PIR (Passive InfraRed), GPS and colour sensors.

By mounting a smartphone onto a helmet, Chennuru et al. captured pictorial life-logging data and supplemented the data with accelerometer data and used language based indexing to organize the data into events [14]. A web based interface was developed to review the recorded data.

The use of life-logging has the potential to address the unmet needs of a PwD, specifically the three needs mentioned above. Data can be retrospectively reviewed to ascertain if a medication was taken, an appointment was made, to whom the wearer interacted with and if they were safe. Nevertheless, this system would be more beneficial to the PwD and indeed their carer, if these lifestyle indicators were monitored in real-time and not retrospectively.

The ability to, in real time, identify social contact is a new area of research. Nevertheless, the AMSSI (Automated Memory Support for Social Interaction) system [15] provides support for recognizing visitors to the user's home i.e. a doctor's visit. The system uses a smartphone and facial recognition to determine the person the wearer is interacting with in addition to monitoring dialogue to determine whether the user requires support. In the event the user requires support the system provides audio feedback to the user. This system is specifically designed to enhance social interaction within the home environment. The benefits of this system are lost if and when the user leaves the home environment. In addition, all potential contacts facial recognition data would need to be captured, analyzed and stored prior or any interaction.

Each of the aforementioned systems has the potential to address individual unmet needs of PwD. The $\mathrm{R}^{3} \mathrm{~S}$ aims to address several of these unmet needs. The difference between these systems and the $\mathrm{R}^{3} \mathrm{~S}$ system are outlined in Table 1 .

TABLE I. A FUNCTIONALITY COMPARISON BETWEEN THE RELATED WORK AND THE $\mathrm{R}^{3}$ S STSTEM

\begin{tabular}{|c|c|c|c|c|c|}
\hline & $\begin{array}{c}\text { Life- } \\
\text { Logging }\end{array}$ & $\begin{array}{c}\text { Geo } \\
\text { Fencing }\end{array}$ & $\begin{array}{c}\text { Social } \\
\text { Interaction }\end{array}$ & Reminiscence & Vitals \\
\hline SenseCam & 凶 & $\square$ & $\square$ & $\square$ & $\square$ \\
\hline Memoto & $\boldsymbol{x}$ & $\square$ & $\square$ & 冈 & $\square$ \\
\hline Autographer & 网 & $\square$ & $\square$ & $\square$ & $\square$ \\
\hline $\begin{array}{c}\text { Chennuru et } \\
\text { al. }\end{array}$ & 凶 & 凶 & $\square$ & 冈 & $\square$ \\
\hline CIRCA & $\square$ & $\square$ & $\square$ & $\boldsymbol{x}$ & $\square$ \\
\hline AMSSI & $\square$ & $\square$ & 政 & $\square$ & $\square$ \\
\hline $\mathrm{R} 3 \mathrm{~S}$ & 冈 & 可 & $\mathbf{x}$ & 凶 & 凶 \\
\hline
\end{tabular}

As shown in Table 1 , the $\mathrm{R} \mathrm{S}$ system offers more functionality suited to addressing the unmet needs of PwD than the other solutions.

In the following Section we outline the technological components of the life-logging functionality of the $\mathrm{R}^{3} \mathrm{~S}$ prototype.

\section{PROTOTYPE}

The life-logging component of the system, miLifeCam, is comprised of two main technology components, an Android smartphone (ZTE Blade) and Sony Smartwatch wearable display. By using the 3.1 megapixel camera, storage capacity, sensors and wireless communication capabilities of the smartphone a life-logging system was developed. The smartphone can be worn around the wearer's neck and can record an image every 30 seconds along with the location, timestamp and accelerometer data.

In addition to the life-logging data, the system can also identify one of a group of persons wearing a predetermined QR code. This code contains a user ID that the system identifies. When a known individual is identified, $\mathrm{R}^{3} \mathrm{~S}$ records 'who' that person is, their relationship to the wearer and the time of the interaction. The smartphone transmits this information to the smartwatch, which in turn vibrates and displays a picture of the person, their name and relationship to the wearer. Figure 1 shows the overall architecture of the system and how its components interact.

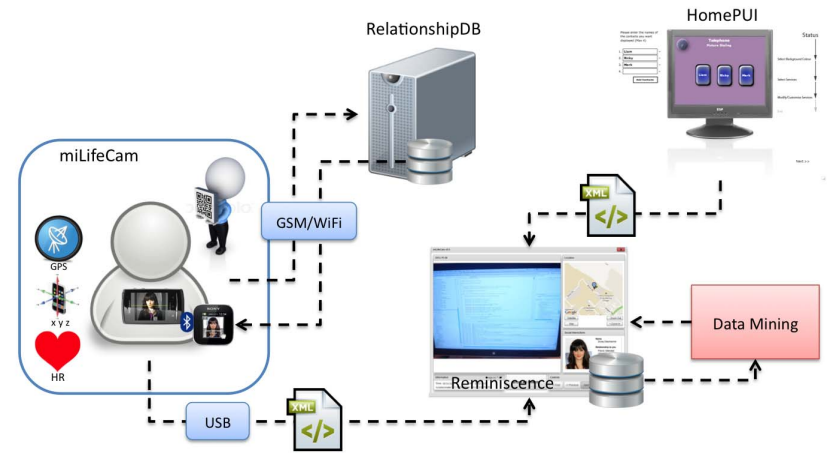

Fig. 1. Architecture of the $\mathrm{R}^{3} \mathrm{~S}$ System detailing the information flow from the wearable life-logger (miLifeCam) to the database (relationshipDB) containg all social contact information. A connection via USB allows the recorded information to be uploaded and displayed on the reminiscence software. The reminiscese software can be personalised in terms of its look and feel by using the HomePUI (Personalised User Interface) tool [16].

A major component of the overall system is the Reminiscence software. This application runs on a desktop PC or laptop and is used to visualize all of the collected life- 
logging data. In addition to reviewing the data, PwDs and carers can use it as a reminiscence tool and annotate and organize the data into events for future reviewing. Details of the Reminiscence software evaluation is not presented in this paper.

An additional function of the system is the recording of the wearer's heart rate. This requires an additional Bluetooth sensor connected to a garment with embedded textile electrodes, shown in Figure 2 (a). By recording the wearer's heart rate it is possible to ascertain physiological features relating to the wearer's location, visual stimulus and social interactions. While this feature may not be beneficial to the wearer in real-time, it can be used to classify events based on the recorded data i.e. increased physical exertion. This component was not part of this evaluation but the authors have used the same methodology in previous work [17], [18].

\section{Evaluation}

The life-logging component of the $\mathrm{R}^{3} \mathrm{~S}$ system (miLifeCam) was evaluated in order to test its general reliability, robustness and accuracy in capturing and recording life-logging data in addition to social interactions. Six healthy participants $(3=$ Male, $3=$ Female) were recruited aged between $24-46$ years of age (Table 2). All of the participants in the study had an above average experience with using technology, smartphones in particular.

The participants were asked to wear the smartphone, attached to a lanyard, around their neck in addition to the smartwatch on their wrist (shown in Figure 2 (b)). The participants carried out their normal daily activities and the system recorded approximately 2.5 hours of data for each participant with an average of 313 images being recorded for each participant.

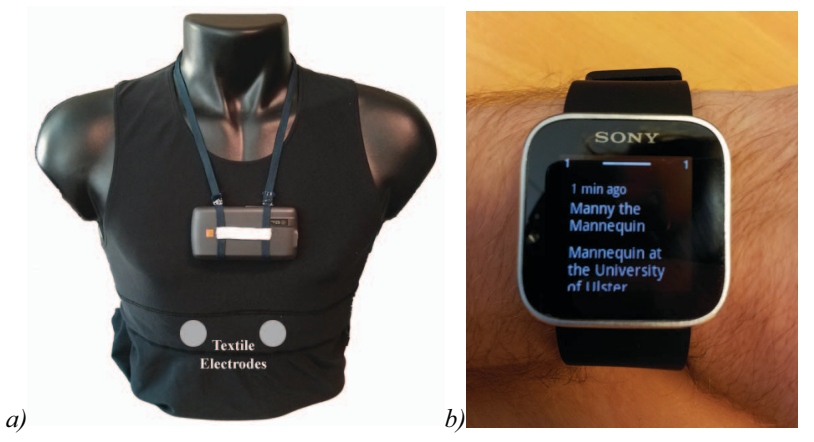

Fig. 2. miLifeCam technology components worn by the participants during the evaluation. a) ZTE Blade smartphone on a lanyard and b) Sony Smartwatch.

Following the recording phase, the participants were asked to review their data using the reminiscence software component (Figure 3). Upon completion of this phase the participants were asked to complete a questionnaire to ascertain the ease of use and effectiveness of the system. In addition to this questionnaire, the participants were asked to score the system using the QUEST (Quebec User Evaluation of Satisfaction with Assistive Technology) questionnaire [19]. The QUEST questionnaire is a 12-item outcome measure that assesses user satisfaction of assistive technologies with two components, Device and Services.
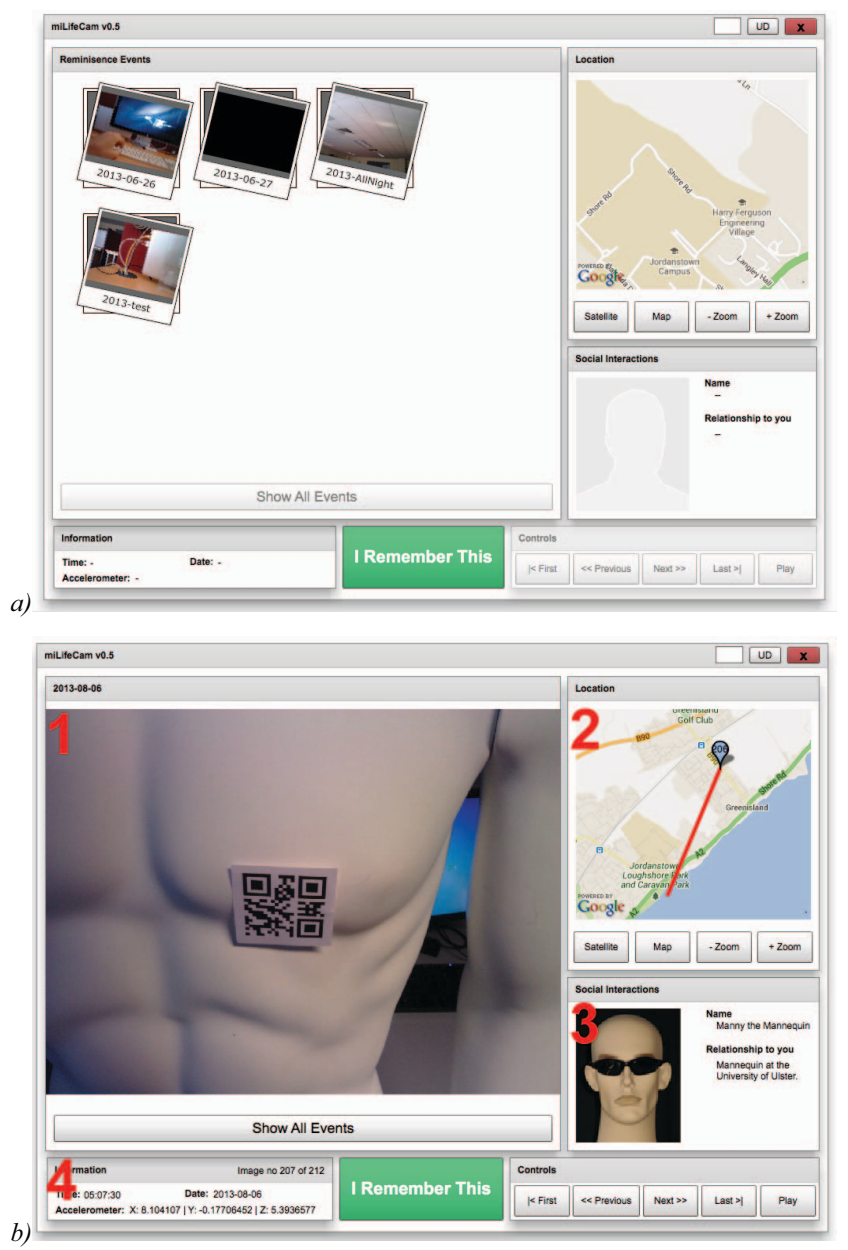

Fig. 3. Screenshots of the reminiscence software developed to review the recorded data from the miLifeCam. This figure shows a) the homescreen with a visual representation of all of the recorded events and $b$ ) the recorded data of a selected event (1) with the location of the image on an interactive map (2), social interactions information (3), accelerometer and time/data information (4).

\section{RESULTS}

In this Section the results of the evaluation of the miLifeCam component of the $\mathrm{R}^{3} \mathrm{~S}$ system are presented. This Section is divided into four Sections each presenting the results of the questionnaires for the a) miLifeCam \& Components, b) Social Interactions, c) QUEST Scores and d) Additional Feedback.

\section{A. miLifeCam and Components}

The participants were asked if they liked using the miLifeCam and how easy they thought it was to use (Figure 4). Additional questions were asked to ascertain the usability, acceptability and aesthetics of the hardware components (smartphone and smartwatch), the results of which are shown in Figures 5 \& 6. 
TABLE II. PARTICIPANT DEMOGRAPHICS

\begin{tabular}{cccc}
\hline \hline Participant \# & Age & Sex & $\begin{array}{c}\text { Technical } \\
\text { expertise }\end{array}$ \\
\hline 1 & 45 & F & Moderate \\
2 & 31 & F & Expert \\
3 & 34 & M & Expert \\
4 & 24 & F & Expert \\
5 & 32 & M & Expert \\
6 & 46 & M & Expert
\end{tabular}

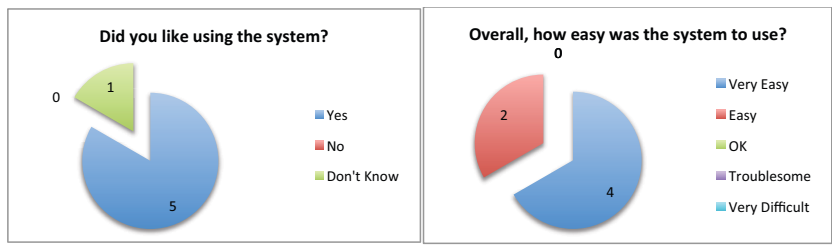

Fig. 4. Charts detailing the qualitative results of the questionnaires for all six participants relating to the overall usability of the miLifeCam system.

As shown in Figure 4, all of the participants liked using the miLifeCam component, with all participants rating the ease of use either 'Easy' or 'Very Easy' to use.

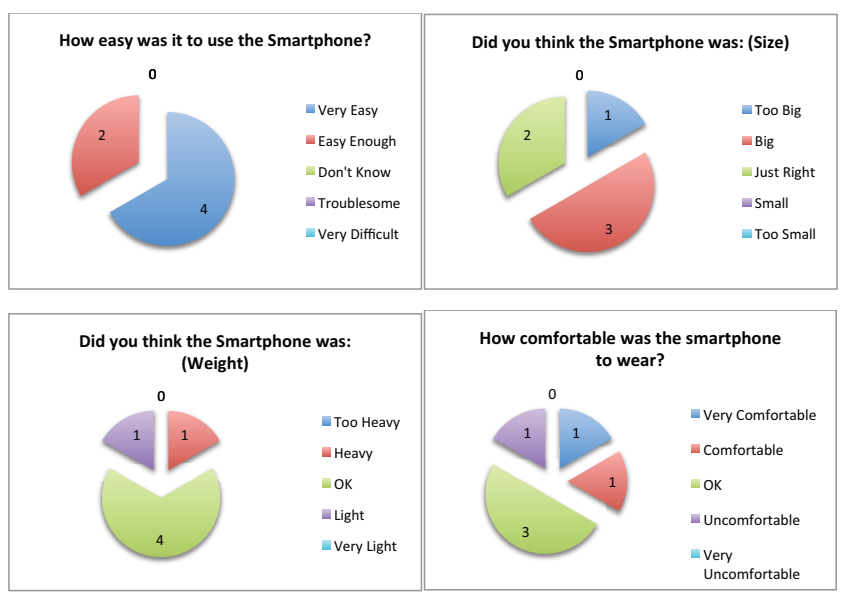

Fig. 5. Charts detailing the qualitative results of the questionnaires for all six participants in relation to the aesthetics of the smartphone used.

The majority of the participants believed that the smartphone (ZTE Blade) was 'big', nevertheless they believed the weight and wearable comfort was acceptable. The software on the smartphone can be deployed on smaller and lighter Android devices such as the Sony Ericsson Xperia X10 Mini.

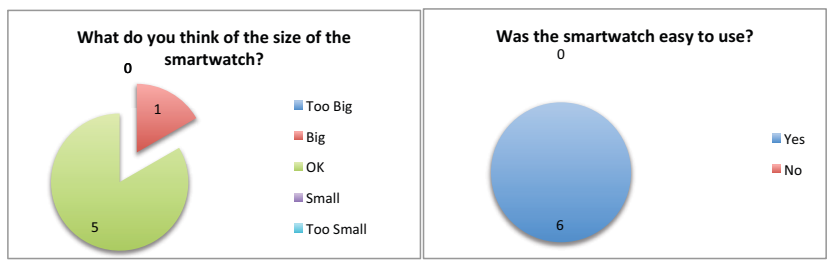

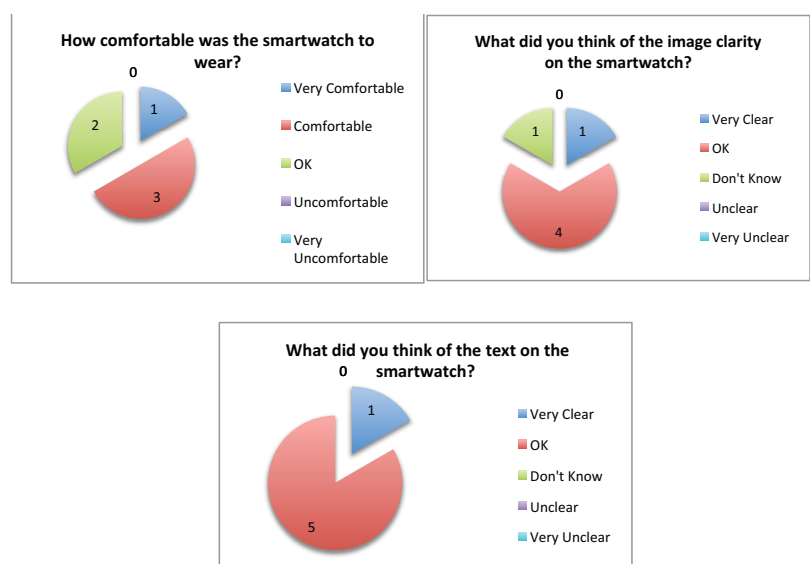

Fig. 6. Chart detailing the qualitative results of the questionnaires for all six participants in relation to the aesthetics and usability of the smartwatch.

Due to the nature of the eventual target cohort, namely PwDs, the miLifeCam and constituent components were developed in such a way as to require as little interaction as possible, however, to carry out their tasks and services in a passive mode and only presenting information to the user when needed.

\section{B. Social Interactions}

During the evaluation, the each participant interacted with a known individual, wearing a QR code, on at least one occasion. During the questionnaires the participants were asked to rate the speed and accuracy of the social interaction detection. Table 3 shows the results of these questions.

TABLE III. PARTICIPANT'S FEEDBACK ON THE NUMBER OF SOCIAL INTERACTIONS THAT TOOK PLACE DURING THE EVALUATIONS, WHERE THEY ABLE TO IDENTIFY THE INTERACTION ON THE SMARTWATCH AND THE PARTICIPANTS THOUGHTS ON ACCURACY.

\begin{tabular}{cccc}
\hline \hline$p \#$ & $\begin{array}{c}\# \text { of } \\
\text { interactions }\end{array}$ & $\begin{array}{c}\text { Identified the interaction on } \\
\text { the smartwatch? }\end{array}$ & Recognition rating? \\
\hline 1 & 1 & Yes & Accurate \\
2 & 2 & Yes & Fast \& Accurate \\
3 & 2 & Yes & Slow \\
4 & 2 & Yes & Accurate \\
5 & 5 & No & Accurate \\
6 & 2 & Yes & Fast \& Accurate
\end{tabular}

As Table 3 shows, the miLifeCam recognition system was able to identify all social interactions with a known individual. Interactions deemed 'fast' and 'accurate' were identified within five seconds of the interaction taking place. 'Slow' interactions took more than five seconds to recognize the interaction. The system was slow to identify the interaction on some occasions but overall performed well and accurately. Not only were the interactions recognized, the real time feedback was sent to the smartwatch and also logged as part of the life-logging service.

\section{QUEST Scores}

QUEST questionnaires were utilized in order to evaluate how satisfied the users were with the assistive device and the related services they experienced. Figure 7 presents the average 
score for each participant in relation to their satisfaction with the assistive technology (miLifeCam). Figure 8 presents the top satisfaction items selected by the participants on the QUEST scoring sheet.

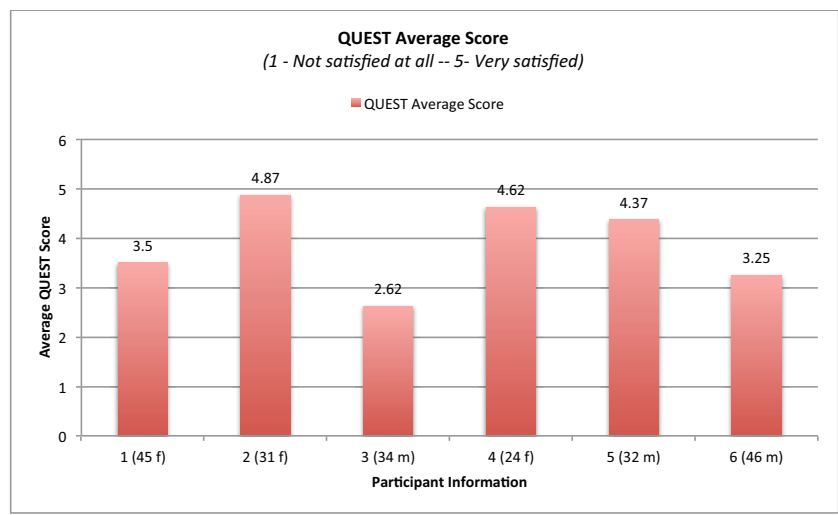

Fig. 7. The average QUEST score relating to participant device satisfaction with $1=$ Not satisfied at all to $5=$ Very Satisfied. The participants ages and gender are also shown.

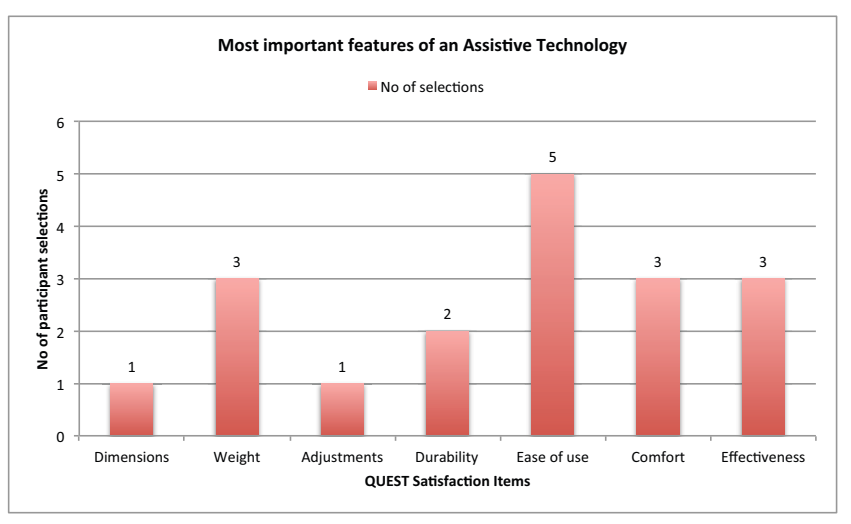

Fig. 8. Detail of the most important features selected by the six participants as part of the QUEST questionnaire. Other options are available but excluded form the figure because they scored 0 .

Items excluded from Figure 8, because that were not selected by any participant were Safety, Service Delivery, Repairs/Servicing, Professional Service and Follow up service all of which are options within the QUEST questionnaire.

\section{Additional Feedback}

The participants were able to make any additional comments they thought necessary on the supplied questionnaires. This feedback ranged from reflection on the comfort of the technology devices, such as the smartwatch "Itched under the strap a little" (Participant 1) to general thoughts such as "the system was easy to use and surprisingly easy to forget about" (Participant 6).

Several of the participants remarked on the questionnaire that they had forgotten that they were wearing the system. They did not, however, specify if they meant the system as a whole or just the smartphone or watch.
As the system was designed with PwD in mind, once the smartphone and watch were on, the user did not have to do anything with the technology. This came through in the feedback from the participants who commented that they "...didn't have to do anything" (Participant 6) and that it was "Simple and Intuitive" (Participant 3) and "...simple to use" (Participant 4).

\section{OBSERVATIONS}

During the review process of the evaluations, where the participants used specially developed reminiscence software to review the captured images, the authors noticed some key images that may be beneficial to the proposed target cohort. It proved useful to track the social interactions the PwD may have, and the locations to which they travelled. It was noted that a number of the participants captured clear images on them eating food and this may be an additional attribute to record and interpret. While this may be fairly inconsequential to the participants in this evaluation, it would be beneficial for carers to 'know' that their mother/father had eaten something that was nutritious. A simple task that may otherwise be overlooked by the PwD.

QR Codes can readily identify social interactions with known individuals. Nevertheless, there are some disadvantages of QR codes, the first being that the individual needs to wear the code, ideally, around the chest area. If the individual is standing at an angle to the wearer, then camera is less likely to identify the code. An ideal solution would be the use of facial recognition processing, similar to that presented in [15].

The use of a smartwatch as a wearable and unobtrusive feedback mechanism is shown to be beneficial, and can be used in a number of differing scenarios. Textual and pictorial information can be delivered to the wearer alerting them to the status of a device, such as messages or calls, as well as other information such as activity levels [17].

\section{CONCLUSIONS}

In this paper we have presented an evaluation of a wearable life-logging system designed for use by PwD. Not only does this system facilitate the capturing of life-logging data in the form of images and location, but also social interaction with known individuals. We have demonstrated that the miLifeCam system performed well, with all participants finding the system easy to use. The system itself recorded all images within the evaluation timeframe and correctly captured and presented any social interactions.

The system could potentially be used to enable PwD to capture their daily activities in image format in addition to supplementary data such as location and social interactions. It will also enable a real-time prompt of social interactions the PwD may have.

Future work will involve the same evaluation procedure but with a target cohort of users aged 55 years old and above. The use of cognitively intact older people in the evaluation of technologies for PwD has been previously investigated [20] and although not perfect, is beneficial when recruitment of $\mathrm{PwD}$ is increasingly difficult. It will also evaluate the system's 
usability on users who have little or no previous experience with smartphone technologies.

\section{ACKNOWLEDGMENT}

The authors would like to acknowledge and thank all of the participants who took part in the study.

\section{REFERENCES}

[1] "CIA World Fact Book." [Online]. Available: https://www.cia.gov/library/publications/the-worldfactbook/geos/xx.html. [Accessed: 09-Jul-2013].

[2] D. H. Paterson and D. E. Warburton, "Physical activity and functional limitations in older adults: a systematic review related to Canada's Physical Activity Guidelines.," The international journal of behavioral nutrition and physical activity, vol. 7, p. 38, Jan. 2010.

[3] K. Kinsell and W. He, "An aging world: 2008," US Census Bureau, International Population Reports, no. June, 2009.

[4] J. Wallace, M. D. Mulvenna, S. Martin, S. Stephens, and W. Burns, "ICT Interface Design for Ageing People and People with Dementia," in Supporting People with Dementia Using Pervasive Health Technologies, Springer London, 2010, pp. 165-188.

[5] "Office for National Statistics, Ageing, "Fastest Increase in the 'Oldest Old'."[Online]. Available: http://www.statistics.gov.uk/cci/nugget.asp?id=949. [Accessed: 09Jul-2013].

[6] "Cost of Care Increases." [Online]. Available: http://www.guardian.co.uk/society/2011/mar/21/care-old-agefunding. [Accessed: 10-Jul-2013].

[7] N. Armstrong, C. Nugent, G. Moore, D. Finlay, and W. Burns, "Inactivity Monitoring for People with Alzheimer's Disease Using Smartphone Technology," in Wireless Mobile Communication and Healthcare, Springer Berlin Heidelberg, 2012, pp. 313-321.

[8] S. Lauriks, A. Reinersmann, H. G. Van Der Roest, F. Meiland, R. Davies, F. Moelaert, M. D. Mulvenna, C. D. Nugent, and R. Dröes, "Supporting People with Dementia Using Pervasive Health Technologies," no. 2007, pp. 37-61, 2010.

[9] A. Sellen and S. Whittaker, "Beyond total capture: a constructive critique of lifelogging," Communications of the ACM, 2010.

[10] "Microsoft SenseCam." [Online]. Available: http://research.microsoft.com/enus/um/cambridge/projects/sensecam/. [Accessed: 09-Jul-2013].
[11] A. R. Doherty, C. J. a Moulin, and A. F. Smeaton, "Automatically assisting human memory: a SenseCam browser." Memory (Hove, England), vol. 19, no. 7, pp. 785-95, Oct. 2011

[12] "Memoto." [Online]. Available: http://memoto.com/. [Accessed: 09Jul-2013].

[13] "Autographer," 2013.2 [Online]. Available: http://www.autographer.com/\#home. [Accessed: 19-Dec-2013].

[14] S. Chennuru, P.-W. Chen, J. Zhu, and J. Zhang, "Mobile Lifelogger - Recording, Indexing, and Understanding a Mobile User's Life," in Mobile Computing, Applications, and Services SE - 15, vol. 76, M. Gris and G. Yang, Eds. Springer Berlin Heidelberg, 2012, pp. 263281.

[15] L. Bellodi and R. Jasinschi, "Dialogue support for memory impaired people," Signal \& Information ..., 2012.

[16] W. P. Burns, C. D. Nugent, P. J. McCullagh, H. Zheng, D. D. Finlay, R. J. Davies, M. P. Donnelly, and N. D. Black, "Personalisation and configuration of assistive technologies.," in Conference proceedings : ... Annual International Conference of the IEEE Engineering in Medicine and Biology Society. IEEE Engineering in Medicine and Biology Society. Conference, 2008, vol. 2008, pp. 3304-7.

[17] W. Burns, C. Nugent, P. McCullagh, D. Finlay, I. Cleland, S. McClean, B. Scotney, and J. McCann, "A smart garment for older walkers," in Impact analysis of solutions for chronic disease prevention and management, 2012, pp. 258-261.

[18] W. P. Burns, C. D. Nugent, P. J. McCullagh, D. D. Finlay, I. Cleland, B. W. Scotney, S. I. McClean, J. McCann, and D. Gueldenring, "Evaluation of a technology enabled garment for older walkers," in Engineering in Medicine and Biology Society (EMBC), 2012 Annual International Conference of the IEEE, 2012, pp. 21002103.

[19] L. Demers, R. Weiss-Lambrou, and B. Ska, "The Quebec User Evaluation of Satisfaction with Assistive Technology (QUEST 2.0): An overview and recent progress," Technology and Disability, vol. 14, no. 3, pp. 101-105, Jan. 2002.

[20] R. Landau, S. Werner, G. K. Auslander, N. Shoval, and J. Heinik, "What do cognitively intact older people think about the use of electronic tracking devices for people with dementia? A preliminary analysis.," International psychogeriatrics / IPA, vol. 22, no. 8, pp. 1301-9, Dec. 2010. 